\title{
WHITEFLIES (HEMIPTERA : ALEYRODIDAE) INFESTED ORCHARDS AND THEIR NATURAL ENEMIES IN EGYPT
}

\author{
ABD-RABOU, SHAABAN AND NOHA AHMED
}

Plant Protection Research Institute, ARC, Dokki, Giza, Egypt

(Manuscript received 12 February 2012)

\begin{abstract}
Whiteflies (Aleyrodidiae: Hemiptera) are the most economic pests infested orchards trees in Egypt. The present work dealt with the whiteflies infested orchards and their natural enemies in Egypt as well as world literature of host plants, distribution and natural enemies of these pests. The results indicated that nine species of whiteflies were recorded infested orchards trees in Egypt as well as 8 new natural enemies associated with these pests. Also constructed key of the nine species of whiteflies was provided. The host plants, distribution and natural enemies were reported. The seasonal abundance of the black citrus whitefly ,Acaudaleyrodes rachipora (Singh) was studied for two successive years from 20102011 on citrus trees in Qalyubyia. The obtained results showed that, the insect population reached maximum during April in first and second years, respectively. Numbers of Encarsia acaudaleyrodis reached maximum during April during the first and second years .There is no predators collected from this location. The seasonal abundance of sycamore whitefly, Aleurolobus marlatti (Quaintance) was studied for two successive years from 2010-2011 on sycamore trees. The obtained results showed that, the insect population reached maximum during May in the first and second years, respectively. Numbers by Encarsia elegans reached maximum during May during the first and the second years. The predator Orius lavigatus reached maximum during May of the first and the second years. The seasonal abundance of the olive whitefly, Aleurolobus olivinus (Silvestri) was studied for two successive years from 2010-2011 on olive trees in El-Arish .The obtained results showed that, the insect population reached maximum during June in first year and May in the second one. Numbers of Encarsia olivina reached maximum during June of the first year and May during the second one. There is no predators collected from this location. The seasonal abundance of the pomegranate whitefly, Siphoninus phillyreae (Haliday) was studied for two successive years from 2010-2011 on pomegranate trees in Assuit. The obtained results showed that, the insect population reached maximum during August in both first and second year. Numbers of Encarsia inaron reached maximum during August of the first year and September during second years .There is no predators collected from this location. Also the same study conducted during two successive years from 2010-2011 on pomegranate trees in Daqahlyia .The obtained results showed that, the insect population reached maximum during August in first and second years. Numbers of the predator Clitostethus arcuatus reached maximum during August of the first year and July during second years .There is no parasitoids collected from this location. During the present work the whitefly Bemisia afer
\end{abstract}


(Priesner \& Hosny) on citrus during 2010-2011 was very rarely in Behira and 47 and 62 individuals only collected during the first ad second years, respectively The numbers of the parasitoid, $E$. mundus was also very rarely, 4 individuals only collected during the period of study.During the present work the whitefly Aleuroclava jasmini, the whitefly Bemisia afer(Priesner \& Hosny) and the cotton whitefly, Bemisia tabaci and their parasitoids were very rarely on citrus during 2010-2011 in Qalyubiya. During the present work the citrus whitefly, Dialeurodes citri (Ashmead) and Japanese bayberry whitefly, Parabemisia myricae (Kuwana) on citrus during 2010-2011 was not recorded and collected during the period of study.

\section{INTRODUCTION}

Egypt is well known for its excellent climate and fertile land. There is also adequate water for irrigation and wide variety of soils, as well as a dynamic human resource. These favorable characteristics permit the cultivation of almost all species of fruit trees known to the world, except those that have high chilling requirements (Mansour,1995). Aleyrodidae (Whiteflies) has a world distribution and comprises about 1556 species in 161 genera (Evans, 2008). Out of them, 22 species were recorded from Egypt (Abd-Rabou, 2011). Whiteflies cause direct damage by feed on plant juices and indirect damage due to secretions of honey dew that lead to closure of the gaps respiratory and the growth of black mold fungi on leaf surfaces .Thus affecting on photosynthesis and biggest threat comes from the transfer of viral diseases (Papayiannis, et. al. 2008). Priesner and Hosny (1934) described the Aleurolobus niloticus Priesner and Hosny ( $A$. maraltti) on various host plant, Bemisia afer (Priesner and Hosny) on Lawsonia alba and Ficus sycmorus, Aleurotrachelus alhagii Priesner and Hosny ( $A$. rachipora) on several leguminous plants, and Siphoninus phillyreae (Haliday) is recorded on pear, apple and pomegranate (Punica granatum). Habib and Farag (1970) described nine species of Aleyrodidae that are common in Egypt, with notes on their host plants and local distribution. Later Abd-Rabou (1996) added newly recorded species to the Egyptian whiteflies; Dialeurodes citri (Ashmead) and Parabemisia myricae (Kuwana) on citrus.

Many Egyptian workers dealt with the natural enemies of whiteflies,e.g. Priesner and Hosny (1940), Hafez et. al. (1979,a), Abd-Rabou and Abou-Setta (1998) , AbdRabou (1999,a) and Abd-Rabou (2006b).

The aim of the present work is to study the host plants, distribution, natural enemies and seasonal abundance of whiteflies infested orchards in Egypt as well as a constructed key of these species in Egypt. 


\section{MATERIALS AND METHODS}

Infested leaves of citrus,olive, pomegranate, sycamore were examined in the field using a pocket magnification lens. Infested leaves and twigs were collected from different host plants and different locations in Egypt during 2010-2011. Identification of whiteflies was done by examining adults in Canada Balsam . Also Infested leaves will be examined in the field, using a pocket lens. The leaves and twigs will be collected and placed separately in paper bags for further examination in the laboratory. Materials will be kept in a well-ventilated container until the emergence of any natural enemies. Identification of natural enemies will be made by examining mounted adults in Hoyers medium . World host plants, distibution and natuarl enemies will be adapted according to Evans, 2008. Abundance of the populations of whiteflies and their natural enemies infested orchard trees were carried out on olive trees during 2010 and 2011 in Assuit, Daqahlyia, Sharqyia, El-Arish, Qalyubyia, . The plant areas selected for these investigations received no chemical control measures for several years. Twenty trees of citrus, olive, pomegranate, sycamore almost similar in age, size, shape and growth condition were randomly chosen for sampling at a month intervals for each location. On each sampling , 30 leaves of citrus and sycamore and 60 leaves of olive and pomegranate were chosen at random. Thereafter, the leaves were kept in a closed paper bags and transferred to the laboratory for further examination and counting. Each leaf was stored in a wellventilated emergence glass tube and monitored daily for parasitoid emergence. Rate of parasitism was determined by dividing the number of emerging parasitoid from each by the number of hosts existing. Predators was counted in filed and transferred to the laboratory for further examination. Simple correlation and regression values were calculated to obtain information about the relationships between the three tested weather factors and the population of the pest and its natural enemies.

\section{RESULTS AND DISCUSSION}

The present list includes 9 species of whiteflies attacking citrus, olive, pomegranate and sycamore trees in Egypt.

\section{List of Whiteflies infested orchards in Egypt}

1. Acaudaleyrodes rachipora (Singh)

2. Aleuroclava jasmini (Takahashi)

3.Aleurolobus marlatti (Quaintance)

4. Aleurolobus olivinus (Silvestri) 
5. Bemisia afer Priesner \& Hosny

6. Bemisia tabaci (Gennadius)

7. Dialeurodes citri (Ashmead)

8. Parabemisia myricae (Kuwana)

9.Siphoninus phillyreae (Haliday)

\section{Key to Whiteflies infested orchards in Egypt}

1. Pupal case black .... 2

. Pupal case pale or yellowish.

4

2. Operculum filling about one quarter of vasiform orifice86, small groups of tuberclelike markings present along median line of abdominal segments, cuticle unicolorous, brown to black, margin regularly toothed but the teeth may be obscured by downcurling Acaudaleyrodes rachipora (Singh) on citrus

- Operculum almost filling vasiform orifice, tracheal pore areas mostly differentiated from margin by a comb

3. Pupal case subcircular, tracheal combs with about six teeth, caudal setae absent Aleurolobus olivinus (Silvestri) on olive

- Pupal case suboval, tracheal combs with three teeth, caudal setae conspicuou Aleurolobus marlatti (Quaintance) on ziziphus

4. Vasiform orifice relatively small, subcircular posterior margin often with small median tubercle. Operculum usually concealing lingula. Inner margins toothed or smooth

Dialeurodes citri (Ashmead) on citrus

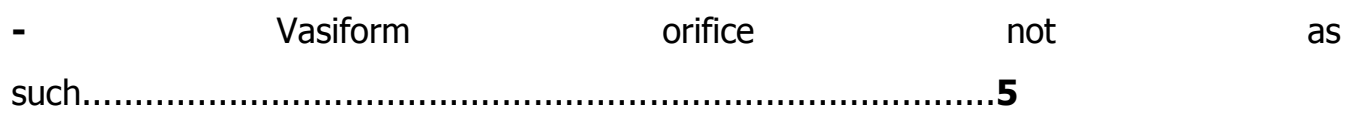

5. First abdominal setea present, abdominal tracheal pore area differentiated by a comb from margin, sculpture of vasiform orifice floor typically with more, smaller, areolae Siphoninus phyllirea on pomegranate

- First abdominal setea absent

.6

6. Vasiform orifice open, operculum subequal in shape, about three-fourth as 'long as and not quite half the length of the orifice, lingula about five-sixth of the orifice, the distal two-fifths enlarged and arrow shaped, thickly setose and terminiating setea floor with transverse ridges distinct, teeth of inner margin present Parabemisia myricae (Kuwana) on citrus

- Vasiform orifice not as such. 
7. Spiracles usually small and subequal in size, somtimes the anterior abdominal spiracles are reduced or absent, margin crenulate. Aleuroclava jasmini (Takahashi) on citrus

- Spiracles not as such 8

8. Vasiform orifice posteriorly with some transverse ridges, antennae with basal spines Bemisia afer Priesner \& Hosny on citrus

-Vasiform orifice posteriorly with tubercles, antennae without basal spines Bemisia tabaci (Gennadius) on citrus

\section{Acaudaleyrodes rachipora (Singh)}

\section{Host Plants}

World: Anacardiaceae: Rhus sp; Annonaceae: Hexalobus monopetalus; Araceae: Anubias sp; Asclepiadaceae:Leptadenia heterophylla; Burseraceae: Commiphora sp; Caprifoliaceae: Sambucus nigra;Combretaceae: Combretum micranthum, Terminalia laxiflora; Euphorbiaceae: Euphorbia pilulifera, Hymenocardia acida; Ricinus communis, Fabaceae: Abrus precatorius,.Acacia arabica, Acacia nilotica, Albizzia odoratissima; Alhagi sp, Bauhinia sp, Cassia auriculata, Cassia fistula, Cassia italica, Cassia sieberiana, Ceratonia siliqua, Cercis siliquastrum, Dalbergia sissoo, Delonix elata, Detarium microcarpum, Dichrostachys glomerata, Inga dulce, Lonchocarpus laxiflorus, Parkia clappertoniana, Prosopis juliflora, Prosopis africana, Prosopis stephaniana, Pterocarpus lucens, Tamarindus indica, Tephrosia apollinea, Tephrosia linearis, Lythraceae: Lawsonia inermis, Moraceae: Ficus sp, Morus alba, Morus nigra; Myrtaceae: Psidium guajava; Ochnaceae: Ochna afzelii, Punicaceae: Punica granatum; Rhamnaceae: Ziziphus spina-christi; Rutaceae: Citrus aurantifolia, Citrus sinensis, Citrus limon; Sapindaceae: Dodonaea viscosa; Tiliaceae: Grewia similis, Verbenaceae: Vitex simplicifolia; Zygophyllaceae: Balanites aegyptiaca; Entrada africana.

Egypt: Anacardiaceae:Rhus albida, Asclepiadaccae:Leptadenia heterophylla, Caprifoliaceae:Sambucus nigra,Euphorbiaceae: Euphorbia cuneata, Leguminosae:Acacia $\quad$ sp.,Acacia $\quad$ nilotica,Acacia $\quad$ tortilis,Alhagi sp.,Alhagi maurorum,Cassia italica, Prosopis sp.,Prosopis spicigera, Prosopis stephaniana,Tephrosia apollinea,Tephrosia leptostachya,Lythraceae, Lawsonia alba,Lawsonia inermis, Myrtaceae: Psidium guajava7, Punicaceae:Punica granatum,Rhamnaceae: Ziziphus spina-christi, Rosaceae:Rosa sp., Rutaceae:Citrus aurantium var. amara, Salvadoraceae:Dodonaea visceosa, Solanaceae:Lycopersicum esculentum, Tiliaceae: Gerwia tenax,Zygophyllaceae:Balanites aegyptiaca . 


\section{Distribution}

World: Cameroon, Canary Islands, Chad, Cyperus, India, Iran, Iraq, Israel, Jordan, Kenya , Liberia, Madagascar, Niger, Nigeria and Saudi Arabia, Sierra Leon, South Africa and Sudan

Egypt: Aswan, Beni-Suef, Cairo, Dakhla Oasis, Daqahliya, Eastern desert, El-Minya, Kafr El-Sheikh, Gharbiya, Minufiya, Qena, Sharqiya, Sohag

\section{Natural enemies:}

World: Parasitoids : Encarsia acaudaleyrodis, Encarsia davidi, Encarsia galilea,Encarsia inaron; Encarsia lutea, Encarsia mineoi, Encarsia sophia, Eretmocerus rajasthanicus, Eretmocerus roseni, Predators : ACARI/Phytoseiidae: Euseius scutalis.

\section{Egypt: Encarsia acaudaleyrodis, Encarsia mineoi}

Abundance: The seasonal abundance of the black citrus whitefly was studied for two successive years from 2010-2011 on Citrus trees in Qalyubyia .The obtained results in Figs ( 1 and 2 ) showed that, the insect population reached maximum during April (15 and 14/ 30 leaves ) in first year and second year, respectively. Numbers by Encarsia acaudaleyrodis reached maximum ( 2 and 2 / 30 leaves ) during April during the first year and second years, respectively .There is no predators collected from this location. Data in Table (1), show that the simple correlation between the population of parasitoids, maximum, minimum temperature, relative humidity and the mean number of the pest were non-significant ( $r=0.20,0.64,0.59$ and 0.33 ), respectively during the 2010 year. Also, results in Table (1), show that the simple regression for changing the population of parasitoids, maximum, minimum temperature, relative humidity and the mean number of the pest were also nonsignificant ( $b=0.19,0.61,0.57$ and 0.27 ), respectively during the 2010 year. Data in Table (2), show that the simple correlation between the population of parasitoids, predators, maximum, minimum temperature, relative humidity and the mean number of the pest were non-significant $(r=0.22,0.61,0.52$ and 0.18$)$, respectively during the 2011 year. Also, results in Table (2), show that the simple regression for changing the population of parasitoids, predators, maximum, minimum temperature, relative humidity and the mean number of the pest were also non-significant $(b=0.18,0.59$, 0.50 and 0.15$)$, respectively during the 2011 year. 
Comment: This species was first recorded in Egypt by Priesner and Hosny (1934) as Aleurotrachelus alhagii Priesner and Hosny . Later Abd-Rabou (1996) recoreded this species infested citrus trees in Egypt.

\section{Aleuroclava jasmini (Takahashi)}

\section{Host Plants}

World: Myrsinaceae: Maesa japonica; Rubiaceae: Jasminum sambac, Rubiaceae: Mitragyna sp.; Rutaceae: Citrus sinensis, Murraya paniculata.

Egypt: Rutaceae: Citrus sinensis

\section{Distribution}

World: China, Guam, Hong Kong, India, Indonesia, Japan, Paraguay, Philippines, Singapore, Taiwan, Thailand and USA.

Egypt: Qalyubiya

\section{Natural enemies}

World: Parasitoids : Encarsia lutea, Encarsia nipponica, Encarsia perflava.

Egypt: Encarsia lutea *

Abundance:During the present work the whitefly Aleuroclava jasmini on citrus during 2010-2011 was very rarely in Behira and 55 and 88 individuals only collected during the first and second years, respectively The numbers of the parasitoid, E. lutea was also very rarely, 8 individuals only collected during the period of study.

Comment: This species reported in Egypt on Citrus by Evans(2008). In the prsent work Encarsia lutea recorded for the first time associted with this species.

\section{Aleurolobus marlatti (Quaintance)}

\section{Host Plants}

World: Amaranthaceae: Amaranthus bilitoides, Apocynaceae: Nerium indicum; Araceae: Colocasia sp; Araliaceae: Hedera nepalensis, Asclepiadaceae: Leptadenia hastata; Bignoniaceae: Steroespermum kunthianum; Bombacaceae: Bombax malabaricum; Boraginaceae: Ehretia aspersa; Capparidaceae: Boscia senegalensis, Capparidaceae: Capparis corymbosa; Daphniphyllaceae: Daphniphyllum macropodum; Ebenaceae: Diospyros mespiliformis; Hymenocardia acida, Glochidion hongkongensis, Mallotus philippensis, Fabaceae: Dalbergia sissoo, Tephrosia purpurea; Liliaceae: Smilax sp; Lythraceae: Lawsonia alba, Lawsonia inermis, Malvaceae: Abutilon foliosum, Malvaceae: Thespesia populnea; Moraceae: Ficus sycomorus, Ficus sp, Morus alba; Myrtaceae: Eucalyptus camalduleis,Oleaceae: Olea cuspidata; Punicaceae: Punica granatum; Rhamnaceae: Paliurus spina-christi, Ziziphus jujube, Ziziphus hysudrica, Ziziphus mauritiana, Ziziphus spina-christi, Ziziphus sp; Rosaceae: 
Rosa indica; Rutaceae: Citrus limmeta, Citrus nobilis, Citrus sinensis, Murraya exotica, Murraya paniculata, Murraya koenigii; Salvadoraceae: Salvadora persicae; Sapindaceae: Dodonaea viscosa, Schleichera oleosa; Ulmaceae: Aphananthe aspera; Verbenaceae: Duranta sp, Verbena officinalis, Gmelina sp; Zygophyllaceae: Balanites aegyptiaca.

Egypt: Lythraceae:Lawsonia inermis, Moraceae:Ficus sycamorus, Rhamnaceae:Ziziphus sp., Ziziphus jujubaSalvadoraceae:Salvadora sp., Salvadora persicae, Dodonaea viscosa, Zygophyllaceae:Balanites sp., Balanites aegyptiaca

\section{Distribution}

World: Iran, Israel, Jordan, Saudi Arabia, Chad, China, Japan, Philippines, Taiwan, India, Malaysia, Java.

Egypt: Aswan, Assiut, Cairo, Dakhla Oasis, Eastern desert

\section{Natural enemies:}

World: Parasitoids: Aphelinidae: Encarsia perflava, Encarsia Iongifasciata, Encarsia davidi,Encarsia elegans, Encarsia ancistrocera, Encarsia lutea, Eretmocerus aleurolobi, Eretmocerus siphonini, Eretmocerus longiscapus, Eretmocerus parasiphonini, Eretmocerus sp. Predators :Coccinellidae: Brumoides suturalis.

Egypt: Encarsia davidi*, Encarsia elegans, Encarsia lutea*, Eretmocerus siphonini*, Eretmocerus sp. *

Abundance: The seasonal abundance of sycamore whitefly , Aleurolobus marlatti was studied for two successive years from 2010-2011 on sycamore trees .The obtained results in Figs ( 3 and 4 ) showed that, the insect population reached maximum during May (973 and 1244/ 60 leaves ) in the first and second years, respectively. Numbers by Encarsia elegans reached maximum during May (47 and 95/ 60 leaves ) during the first year and the second year, respectively. The predator Orius lavigatus reached maximum during during May (22 and 25/ 60 leaves ) during the first year and the second year, respectively.

Data in Table (3), show that the simple correlation between the population of parasitoids, predators, maximum, minimum temperature, \%relative humidity and the mean number of the pest were significant and highly significant $(r=0.71,0.60,0.91$, 0.89 and 0.74 ), respectively during the 2010 year. Also, results in Table (3), show that the simple regression for changing the population of parasitoids, predators, maximum, minimum temperature, \%relative humidity and the mean number of the pest were also significant and highly significant $(b=0.69,0.55,0.90,0.85$ and 0.71 ), respectively during the 2010 year. Data in Table (4), show that the simple correlation 
between the population of parasitoids, predators, maximum, minimum temperature, $\%$ relative humidity and the mean number of the pest were significant and highly significant ( $r=0.84,0.65,0.93,0.91$ and 0.78$)$, respectively during the 2011 year. Also, results in Table (4), show that the simple regression for changing the population of parasitoids, predators, maximum, minimum temperature, \%relative humidity and the mean number of the pest were also significant and highly significant $(b=0.81$, $0.61,0.94,0.93$ and 0.76$)$, respectively during the 2011 year.

Comment: Abd-Rabou (2011) cited this species by the name in Egypt. During the this work, the parasitoids, Encarsia davidi, Encarsia lutea and Eretmocerus siphonini and Eretmocerus sp. Recorded associted with this whitefly species for the fist time in Egypt.

\section{Aleurolobus olivinus (Silvestri)}

\section{Host Plants}

World: Oleaceae: Olea sp, Phillyrea angustifolia.

Egypt:Oleaceae: Olea sp.,

Distribution:

World: China, Cyperus, France, Israel, Italy, Morocco, Spain.

Egypt: El-Arish and Fayoum

\section{Natural enemies}

World: Parasitoids : Aphelinidae: Encarsia arabica, Encarsia elegans, Encarsia olivina; Platygastridae: Amitus minervae

\section{Egypt: Encarsia elegans*, Encarsia olivina}

Abundance: The seasonal abundance of the olive whitefly was studied for two successive years from 2010-2011 on olive trees in El-Arish.The obtained results in Figs ( 5 and 6 ) showed that, the insect population reached its maximum during June (33/ 60 leaves ) in first year and May ( 38/ 60 leaves ) in the second year. Numbers by Encarsia olivina reached maximum during June during the first year ( $7 / 60$ leaves) and during May during second years (11 / 60 leaves ). There is no predators collected from this location. Data in Table (5), show that the simple correlation between the population of parasitoids, maximum, minimum temperature, relative humidity and the mean number of the pest were non-significant $(r=0.24,0.74,0.71$ and 0.69), respectively during the 2010 year. Also, results in Table (5), show that the simple regression for changing the population of parasitoids, predators, maximum, minimum temperature, relative humidity and the mean number of the pest were also non-significant $(b=0.21,0.71,0.68$ and 0.65$)$, respectively during the 2010 year. 
Data in Table (6), show that the simple correlation between the population of parasitoids, maximum, minimum temperature, relative humidity and the mean number of the pest were non-significant $(r=0.28,0.70,0.69$ and 0.61$)$, respectively during the 2010 year. Also, results in Table (6), show that the simple regression for changing the population of parasitoids, maximum, minimum temperature, relative humidity and the mean number of the pest were also non-significant $(b=0.24,0.68$, 0.62 and 0.60 ), respectively during the 2010 year.

Comment: This species recorded for the first time in Egypt by Abd-Rabou (1996). Encarsia elegans recorded here for the first time associted with this whitefly species.

\section{Bemisia afer (Priesner \& Hosny)}

\section{Host Plants}

World: Anacardiaceae: Lannea shimperi, Annonaceae: Annona senegalensis, Hexalobus monopetalus, Pistacia mutica; Apocynaceae: Nerium oleander, Bignoniaceae: Steroespermum kunthianum; Bixaceae: Cochlopermum tinctorium; Bombacaceae: Bombax costatum; Burseraceae: Commiphora pedunculata; Celastraceae: Maytenus senegalensis, Clethra arborea, Clethra sp; Caprifoliaceae: Lonicera sp; Combretaceae: Anogeissus leiocarpus, Combretum confertum, Combretum glutinosum, Combretum hypopilinum, Combretum molle; Euphorbiaceae: Antidesma venosum, Bridelia ferruginea, Hymenocardia acida, Manihot esculenta, Ricinus communis, Fabaceae: Acacia nilotica, Adenodolichos paniculatus, Albizia sp, Burkea africana, Cassia siamea, Daniellia olivera, Detarium microcarpum, Dichrostachys glomerata, Entada africana, Isoberlinia doka, Neorautanenia pseudopachyrhiza, Piliostigma thonningii, Pterocarpus lucens, Robinia pseudoacacia, Terminalia laxiflora; Lauraceae: Laurus nobilis, Liliaceae: Smilax aspera; Loganiaceae: Strychnos spinosa; Lythraceae: Lawsonia inermis, Malvaceae: Gossypium hirsutum, Gossypium sp, Hibiscus asper, Malva parviflora; Moraceae: Ficus nitida, Ficus sycamorus, Myrtaceae: Psidium guajava; Papaveraceae: Bocconia frutescens, Rhamnaceae: Ziziphus spina-christi; Rosaceae: Rosa chinensis, Rosa hybrida, Rosa multiflora, Rosa sp, Spiraea cantonensis, Rubiaceae: Crossopoteryx febrifuga, Gardenia triacantha, Nauclea latifolia; Rutaceae: Citrus aurantium, Citrus limon, Citrus limonia, Citrus sinensis, Clausena anisata; Salicaceae: Salix subserrata; Sapindaceae: Allophylus africanus, Paullinia pinnata; Solanaceae: Solanum muricatum; Urticaceae: Gesnouinia arborea, Urtica dioica.

Egypt: Leguminosae:Acacia nilotica, Albizia sp.Lythraceae:Lawsonia alba, Lawsonia inermis, Moraceae:Ficus nitida, Ficus sycamorus, Rhamnaceae:Ziziphus 
spinachristi,Rutaceae:Citrus limonum ,Citrus sinensis, Saliceaceae:Salix subserrata, Verbenaceae: Lantana camara

\section{Distribution}

World: Australia Brazil, Cameroon,Chad,China , Congo, France,Guinea,India; Iran,Ivory Coast Israel, Kenya, Korea , Italy, Madagascar; Mulawi; New Guinea, Niger; Nigeria;Pakistan;Sicily, Sierre Leon;Spain, South Africa, Sudan, Uganda,Zaire

Egypt:Assiut,Aswan , Beheira,El-Minya,Qalyubiya,Sharqiya

\section{Natural enemies}

World: Parasitoids : Hymenoptera/Aphelinidae: Cales noacki, Encarsia galilea, Encarsia levadicola, Encarsia melanostoma, Encarsia noahi, Encarsia pergandiella, Encarsia silvestrii, Encarsia sophia, Eretmocerus mundus, Eretmocerus roseni, Eretmocerus sp; Eulophidae: Euderomphale bemisiae, Euderomphale cortinae, Euderomphale gomer. Predators : Coleoptera; Coccinellidae: Serangium parcesetosum.

\section{Egypt: Encarsia galilea*, Eretmocerus mundus}

Abundance:During the present work the whitefly Bemisia afer on citrus during 2010-2011 was very rarely in Behira and 47 and 62 individuals only collected during the first and second years, respectively The numbers of the parasitoid, E. mundus was also very rarely, 4 individuals only collected during the period of study.

Comment:This species was first recorded as a new economic pest in Egypt by AbdRabou (2006). During this work, the parasitoid, Encarsia galilea recorded associated with this whitefly species for the fist time in Egypt.

\section{Bemisia tabaci (Gennadius)}

\section{Host Plants}

World: Over 250 host plants in many different families.

Egypt: Abd-Rabou and Simmons (2011) recorded and collected 118 species of plants in 79 genera belonging to 28 families.

\section{Distribution}

World: Worldwide

Egypt: Alexandria, Asyut, Aswan, Behira, Beni-Suif, Cairo, Daqhilya, Demyata, ElDakhala, El-Kharga, El-Minia, Faiyum, Gharbiya, Giza, Ismailia, Kafer El-Shikh, Matruh, Menofia, Northern Sinai, Port Said, Qalyubiya, Qena, Red Sea, Sharqiya, Sohag, Southern Sinai, and Suez. 


\section{Natural enemies}

World: Parasitoids : About 60 species of Parasitic Hymenoptera (Evans 2008)

Predators : About 180 species of different orders and families (Evans 2008).

\section{Egypt:}

Abundance:During the present work the cotton whitefly on citrus during 2010-2011 was very rarely in Qalyybiya and 16 individuals only collected during the period of study.

Comment: This pest is not economic on orchards trees in Egypt .

\section{Dialeurodes citri (Ashmead)}

\section{Host Plants}

World:Anacardiaceae: Cotinus coggyria; Apocynaceae: Allamanda neriffolia, Nerium oleander, Aquifoliaceae: Ilex sp, Ilex $x$ attenuata; Araliaceae: Aralia sp, Fatsia sp, Hedera formosana, Hedera helix, Schefflera arboricola; Arecaceae: Sabal megacarpa; Bignoniaceae: Tecoma radicans, Boraginaceae: Ehretia sp; Caprifoliaceae: Lonicera japonica, Viburnum odoratissimum, Viburnum sp, Viburnum suspensum, Viburnum tinus, Ebenaceae: Diospyros khaki, Diospyros sp, Diospyros virginiana; Ericaceae: Rhododendron sp; Euphorbiaceae: Bischofia javanica, Glochidion hongkongense, Glochidion sp, Ricinus communis, Fabaceae: Glycine max, Fagaceae: Lithocarpus sp, Quercus aquatica, Quercus nigra; Hamamelidaceae: Distylium racemosus, Lauraceae: Machilus sp, Persea sp; Liliaceae: Smilax sp; Lythraceae: Lagerstroemia indica; Magnoliaceae: Magnolia coco, Magnolia fuscata, Magnolia sp, Magnolia soulangiana; Malpighiaceae: Hiptage madablota; Hiptage benghalensis, Meliaceae: Melia azedarach; Moraceae: Ficus altissima, Ficus macrophylla, Ficus nitida, Maclura aurantiaca; Myrsinaceae: Ardisia humilis, Ardisia sieboldi; Myrtaceae: Eugenia jambos, Eugenia sp, Myrtus communis, Myrtus lagerstroemia, Psidium guajava; Nyssaceae: Nyssa ogeche; Oleaceae: Chionanthus retusus, Chionanthus virginicus, Forsythia sp, Fraxinus lanceolata, Jasminum arborescens, Jasminum frutescens, Jasminum nitidum, Jasminum odoratissimum, Jasminum sambac, Ligustrum amurense, Ligustrum ibota, Ligustrum japonicum, Ligustrum lucidum, Ligustrum ovalifolium, Ligustrum sinense, Ligustrum sp, Ligustrum vulgare, Osmanthus americanus, Osmanthus fragrans, Osmanthus heterophyllus, Syringa sp, Syringa vulgaris, Proteaceae: Helicia sp; Punicaceae: Punica granatum; Rosaceae: Cerasus sp. Crataegus laevigata, Laurocerasus caroliniana, Malus sp, Prunus caroliniana, Prunus caroliniana, oPrunus laurocerasus, Pyracantha coccinea, Pyracantha koidzumii, Pyrus sp, Rubus sp, Rubiaceae: Cephalanthus occidentalis, Rubiaceae: Cephalanthus sp, Coffea arabica, 
Gardenia augusta, Gardenia florida, Gardenia jasminoides, Gardenia radicans, Gardenia sp, Hedera helix, Jasminum fruticans, Wendlandia glabrata, Rutaceae: Choisya ternata, Citrus aurantifolia, Citrus aurantium, Citrus aurantium var formosanus, Citrus bigaradia, Citrus medica, Citrus ponki, Citrus sinensis, Citrus sp, Citrus nobilis, Citrus paradisi, Citrus tangelo, Fortunella japonica, Poncirus trifoliata, Severinia buxifolia, Citrofortunella microcarpa, Zanthoxylum clava-herculis, Sabiaceae: Meliosma rigida; Sapindaceae: Sapindus sp. Simaroubaceae: Ailanthus glandulosa; Staphyleaceae: Turpinia formosana;Theaceae: Camellia japonica; Theaceae: Camellia sinensis var. sinensis, Vitaceae: Ampelopsis tricuspidata.

Egypt: Citrus spp.

\section{Distribution}

World: Afghanistan, Bahamas, Bermuda, China, Cuba, Dominican Republic, El Salvador, France, Greece , Guam, Haiti, Honduras, Hong Kong, India, Iran, Italy, Japan, Korea, Macau, Mexico, Pakistan, Panama, Philippines, Portugal, Puerto Rico, Sicily, Taiwan, Thailand, Turkey, USA .

Egypt: Behira, Daqhilya, Qalyubiya, Sharqiya,

\section{Natural enemies}

World: Parasitoids - HYMENOPTERA/Aphelinidae: Encarsia abundantia, Encarsia armata, Encarsia citrella, Encarsia citri, Encarsia citrofila, Encarsia formosa, Encarsia lahorensis, Encarsia longivalvula, Encarsia magnivena, Encarsia protransvena, Encarsia sophia, Encarsia sp, Encarsia strenua, Encarsia strenua group, Encarsia tabacivora, Encarsia tricolor, Encarsia variegata, Eretmocerus sp, Eulophidae: Euderomphale sp. Predators - ACARI/Phytoseiidae: Amblyseius eharai, Amblyseius largoensis, Euseius stipulatus, Typhlodromus sp; COLEOPTERA/Coccinellidae: Adonia variegata, Brumoides suturalis, Catana chapini, Chilocorus bipustulatus, Chilocorus stigma, Clitostethus arcuatus, Cryptognatha flaviceps, Cycloneda sanguinea, Delphastus catalinae, Delphastus pallidus, Delphastus pusillus, Delphastus sp, Didion punctatum, Diomus roseicollis, Exochomus quadripustulatus, Menochilus sexmaculatus, Nephaspis oculatus, Olla v-nigrum, Scymnus sp, Serangium flavescens, Serangium parcesetosum, Stethorus gilvifrons, Verania cardoni; DIPTERA/Dolichopodidae: Condylosty/us patibulatus; HEMIPTERA/ Lygeidae: Geocoris sp, Campylomma diversicornis, Deraeocoris pallens, NEUROPTERA/Chrysopidae: Chrysopa sp, Chrysopa vulgaris, Chrysoperla carnea; Coniopterygidae: Conwentzia hagani, Conwentzia psociformis, Semidalis aleyrodiformis, Hemerobiidae: Genus? sp; THYSANOPTERA/ Phlaeothripidae: Aleurodothrips fasciapennis. 


\section{Egypt: Encarsia lahorensis}

Abundance: During the present work the citrus whitefly on citrus during 2010-2011 was not recorded and collected during the period of study.

Comment: This species recorded for the first time in Egypt by Abd-Rabou (1996).

Abd-Rabou (1999) also introduced the parasitoid E. lahorensis from USA for controlling $D$. citri. Also, this parasitoid is established and may be considered an effective antagonist of $D$. Citri.

\section{Parabemisia myricae (Kuwana)}

\section{Host Plants}

World: Asteraceae: Lactuca sp; Cyperaceae: Cyperus papyrus, Ebenaceae: Diospyros khaki; Elaeocarpaceae:Elaeocarpus serratus; Ericaceae: Rhododendron sp; Fagaceae: Quercus serratus, Juglandaceae:Engelhardtia roxburghiana; Lauraceae: Cryptocarya sp,Machilus sp, Persea americana; Moraceae:Ficus carica, Morus alba, Morus sp; Myricaceae: Myrica rubra; Myrsinaceae: Maesa japonica;Myrtaceae: Psidium guajava; Olacaceae: Schoepfia schreberi; Polygonaceae: Coccoloba krugii, Rosaceae: Prunus mume, Prunus persica, Prunus triflora; Rubiaceae: Chiococca alba, Gardenia augusta, Gardenia sp, Gardenia thunbergia; Rutaceae: Citrus aurantifolia, Citrus aurantium, Citrus bigaradia, Citrus limon, Citrus sinensis, Citrus $x$ nobilis, Murraya koenigii, Poncirus trifoliata, Wendlandia thrysoidea, X Citrofortunella microcarpa; Salicaceae: Salix babylonica.

\section{Egypt: Citrus spp.}

\section{Distribution}

World: China, Egypt, India , Iran, Israel, Italy, Japan, Morocco, Spain, Taiwan Turkey, Venezuela, USA.

Egypt: Gharbiya, Kafer El-Shikh, Qalyubiya, Sharqiya.

\section{Natural enemies}

World: Parasitoids : Hymenoptera/ Aphelinidae: Encarsia azimi, Encarsia collecta, Encarsia hispida, Encarsia lutea, Encarsia meritoria, Encarsia perflava, Encarsia protransvena, Encarsia pseudocitrella, Encarsia sophia, Encarsia strenua, Eretmocerus debachi, Eretmocerus furuhashii ;Platygastridae: Amitus sp.Predators :Acari/Phytoseiidae: Amblyseius swirski, Euseius scutalis, Iphiseiodes degenerans, Typhlodromus athiasae; Coleoptera/ Coccinellidae: Adonia variegata, Chilocorus bipustulatus, Clitostethus arcuatus, Coelophora pupillata, Exochomus quadripustulatus, Hippodamia sp, Oenopia conglobata, Scymnus near pallidicollis, Serangium parcesetosum; Coleoptera/ Nitidulidae: Cybocephalus binotatus; Diptera/ 
Syrphidae: Genus? sp; Hemiptera/ Anthocoridae: Genus? Sp; Miridae: Deraeocoris pallens, Neuroptera/Chrysopidae: Chrysoperla carnea; Coniopterygidae:Conwentzia hagani, Conwentzia psociformis, Semidalis aleyrodiformis, Hemerobiidae: Sympherobius

\section{Egypt:Aphelinidae: Encarsia lutea, Platygastridae: Amitus sp}

Abundance: During the present work the citrus whitefly on citrus during 2010-2011 was not recorded and collected during the period of study.

Comment: This species recorded for the first time in Egypt by Abd-Rabou (1996). Abd-Rabou (1998) recorded Encasia lutea as a parasitoid associated with this pest.

\section{Siphoninus phillyreae (Haliday)}

\section{Host Plants}

World: Araliaceae: Hedera canariensis, Fabaceae: Afzelia sp; Myrtaceae: Psidium guajava. Oleaceae: Fraxinus americana, Fraxinus excelsior, Fraxinus syriacata, Fraxinus sp, Olea chrysophylla, Olea europaea, Phillyrea latifolia; Punicaceae: Punica granatum; Rhamnaceae: Rhamnus alaternus, Ziziphus spina-christi; Rosaceae: Crataegus mollis, Crataegus monogyna, Crataegus microphylla, Crataegus sp, Cydonia oblonga, Malus sp, Rosaceae: Mespilus sp, Prunus persica, Pyrus communis, Pyrus malus, Pyrus sativa, Pyrus sp; Rutaceae: Citrus aurantifolia, Citrus sp; Ulmaceae: Ulmus carpinifolia.

\section{Egypt}

\section{Distribution}

World: Australia, Bulgaria, Cameroon , Corsica, Cyprus, England, Egypt, Finland, France, Germany, Greece, Hungary, Iran, Ireland, Israel, Italy, Java , Jordan, Mexico , Peru, Spain, Syria, Sudan, Taiwan, Venezuela, USA, USSR, Yugoslavia, Zaire.

Egypt:Assuit, Behira, Northern Sinai, Southern Sinai.

\section{Natural enemies:}

World: Parasitoids : Hymenoptera: Aphelinidae: Encarsia davidi, Encarsia dichroa, Encarsia galilea, Encarsia gautieri, Encarsia hispida, Encarsia inaron, Encarsia near brasiliensis, Encarsia punicae, Encarsia siphonini, Encarsia strenua, Eretmocerus siphonini. Predators : COLEOPTERA/Coccinellidae: Clitostethus arcuatus, Menochilus sexmaculatus, Menochilus sp, Scymnus smithianus, DIPTERA/ Drosophilidae: Acletoxenus formosus, Acletoxenus indicus, Acletoxenus sp; Empididae: Drapetis ghesquierei.

\section{Egypt: Encarsia inaron. Predators: Clitostethus arcuatus}

Abundance: The seasonal abundance of the pomegranate whitefly was studied for two successive years from 2010-2011 on pomegranate trees in Assuit .The obtained 
results in Figs ( 7 and 8 ) showed that, the insect population reached maximum during August (1850/ 60 leaves ) in first year and August ( 1401/ 60 leaves ) in the second year. Numbers by Encarsia inaron reached maximum during August during the first year (135 / 60 leaves ) and during September during second years (101 / 60 leaves ) .There is no predators collected from this location. Data in Table (7), show that the simple correlation between the population of parasitoids, maximum, minimum temperature, \%relative humidity and the mean number of the pest were significant and non-significant ( $r=0.66,0.79,0.71$ and 0.62 ), respectively during the 2010 year. Also, results in Table (7), show that the simple regression for changing the population of parasitoids, predators, maximum, minimum temperature, \%relative humidity and the mean number of the pest were also significant and non-significant $(b=0.72$, $0.75,0.69$ and 0.61 ), respectively during the 2010 year. Data in Table (8), show that the simple correlation between the population of parasitoids, predators, maximum, minimum temperature, \%relative humidity and the mean number of the pest were significant and non-significant ( $r=0.71,0.73,0.68$ and 0.60$)$, respectively during the 2011 year. Also, results in Table (8), show that the simple regression for changing the population of parasitoids, predators, maximum, minimum temperature, \%relative humidity and the mean number of the pest were also significant and non-significant ( $b$ $=0.66,0.70,0.64$ and 0.58$)$, respectively during the 2011 year.

Also the same study conducted two successive years from 2010-2011 on pomegranate trees in Daqahlyia .The obtained results in Figs (9 and 10) showed that, the insect population reached maximum during August (425/ 60 leaves ) in first year and August ( $280 / 60$ leaves ) in the second year. Numbers by the predator Clitostethus arcuatus reached maximum during August during the first year ( 7 / 60 leaves ) and during July during second years (4 / 60 leaves ). There is no parasitoids collected from this location.

Data in Table (9), show that the simple correlation between the population of predators, maximum, minimum temperature, \%relative humidity and the mean number of the pest were significant and non-significant $(r=0.42,0.70,0.61$ and 0.55), respectively during the 2010 year. Also, results in Table (9), show that the simple regression for changing the population of predators, maximum, minimum temperature, \%relative humidity and the mean number of the pest were also significant and non-significant $(b=0.33,0.67,0.59$ and 0.52$)$, respectively during the 2010 year.Data in Table (10), show that the simple correlation between the population of predators, maximum, minimum temperature, \%relative humidity and the mean number of the pest were significant and non-significant $(r=0.40,0.81$, 
0.72 and 0.68 ), respectively during the 2011 year. Also, results in Table (10), show that the simple regression for changing the population of predators, maximum, minimum temperature, \%relative humidity and the mean number of the pest were also significant and non-significant $(b=0.37,0.82,0.70$ and 0.63$)$, respectively during the 2011 year.

Comment: Abd-Rabou and Abou-Setta (1998) recorded also 7 parasitoid species associated with $S$. phillyreae. They mentioned that the parasitoid $E$. inaron was the dominant parasitoid of $S$. phillyreae in Giza and Assiut with average parasitism rates of 38 and 36.5, respectively. During the present work we collected only Encarsia inaron from Assiut. Abd-Rabou (2006a) recorded this preadtor for the first time asssociated with this pest.

Table 1. Simple correlation and regression values of the population dynamics of Acaudaleyrodes rachipora and its parasitoids and predators on citrus trees in Qalyubyia Governorate during 2010 season.

\begin{tabular}{|l|c|c|c|c|}
\hline Variable & $\begin{array}{c}\text { Simple } \\
\text { correlation "r" }\end{array}$ & Probability "P" & Regression & Probability "P" \\
\hline Encarsia acaudaleyrodis & 0.20 & Ns & 0.19 & Ns \\
\hline Maximum & 0.64 & $*$ & 0.61 & $*$ \\
\hline Minimum & 0.59 & $*$ & 0.57 & $*$ \\
\hline R.H. \% & 0.33 & Ns & 0.27 & Ns \\
\hline
\end{tabular}

Table 2. Simple correlation and regression values of the population dynamics of Acaudaleyrodes rachipora and its parasitoids and predators on citrus trees in Qalyubyia Governorate during 2011 season.

\begin{tabular}{|l|c|c|c|c|}
\hline Variable & $\begin{array}{c}\text { Simple } \\
\text { correlation "r" }\end{array}$ & Probability "P" & Regression & Probability "P" \\
\hline Encarsia acaudaleyrodis & 0.22 & Ns & 0.18 & Ns \\
\hline Maximum & 0.61 & $*$ & 0.59 & $*$ \\
\hline Minimum & 0.52 & $*$ & 0.50 & $*$ \\
\hline R.H. \% & 0.18 & Ns & 0.15 & Ns \\
\hline
\end{tabular}


Table 3. Simple correlation and regression values of the population dynamics of Aleurolobus marlatti and its parasitoids and predators on olive trees in Sharqyia Governorate during 2010 season.

\begin{tabular}{|l|c|c|c|c|}
\hline Variable & Simple correlation "r" & Probability "P" & Regression & Probability "P" \\
\hline Encarsia elegans & 0.71 & $*$ & 0.69 & $*$ \\
\hline Orius lavigatus & 0.60 & $*$ & 0.55 & $*$ \\
\hline Maximum & 0.91 & $* * *$ & 0.90 & $* * *$ \\
\hline Minimum & 0.89 & $* * *$ & 0.85 & $* * *$ \\
\hline R.H. \% & 0.74 & $* *$ & 0.71 & $* *$ \\
\hline
\end{tabular}

Table Simple correlation and regression values of the population dynamics of 4. Aleurolobus marlatti and its parasitoids and predators on olive trees in Sharqyia Governorate during 2011 season.

\begin{tabular}{|l|c|c|c|c|}
\hline Variable & Simple correlation "r" & Probability "P" & Regression & Probability "P" \\
\hline Encarsia elegans & 0.84 & $* *$ & 0.81 & $* *$ \\
\hline Orius lavigatus & 0.65 & $*$ & 0.61 & $*$ \\
\hline Maximum & 0.93 & $* * *$ & 0.94 & $* * *$ \\
\hline Minimum & 0.91 & $* * *$ & 0.93 & $* * *$ \\
\hline R.H. \% & 0.78 & $* *$ & 0.76 & $* *$ \\
\hline
\end{tabular}

Table 5. Simple correlation and regression values of the population dynamics of Aleurolobus olivinus and its parasitoids and predators on olive trees in Al Arish Governorate during 2010 season.

\begin{tabular}{|l|c|c|c|c|}
\hline \multirow{2}{*}{ Variable } & $\begin{array}{c}\text { Simple } \\
\text { correlation "r" }\end{array}$ & Probability "P" & Regression & Probability "P" \\
\hline Encarsia olivina & 0.24 & Ns & 0.21 & Ns \\
\hline Maximum & 0.74 & $*$ & 0.71 & $*$ \\
\hline Minimum & 0.71 & $*$ & 0.68 & $*$ \\
\hline R.H. \% & 0.69 & $*$ & 0.65 & $*$ \\
\hline
\end{tabular}


Table 6. Simple correlation and regression values of the population dynamics of Aleurolobus olivinus and its parasitoids and predators on olive trees in $\mathrm{Al}$ Arish Governorate during 2011 season.

\begin{tabular}{|l|c|c|c|c|}
\hline Variable & Simple correlation "r" & Probability "P" & Regression & Probability "P" \\
\hline Encarsia olivine & 0.28 & Ns & 0.24 & Ns \\
\hline Maximum & 0.70 & $*$ & 0.68 & $*$ \\
\hline Minimum & 0.69 & $*$ & 0.62 & $*$ \\
\hline R.H. \% & 0.61 & $*$ & 0.60 & $*$ \\
\hline
\end{tabular}

Table 7. Simple correlation and regression values of the population dynamics of Siphoninus phillyreae and its parasitoids and predators on pomegranate trees in Assuit Governorate during 2010 season.

\begin{tabular}{|l|c|c|c|c|}
\hline Variable & $\begin{array}{c}\text { Simple } \\
\text { Correlation "r" }\end{array}$ & Probability "P" & Regression & Probability "P" \\
\hline Encarsia inaron & 0.66 & $*$ & 0.72 & $*$ \\
\hline Maximum & 0.79 & $* *$ & 0.75 & $* *$ \\
\hline Minimum & 0.71 & $* *$ & 0.69 & $* *$ \\
\hline R.H. \% & 0.62 & $*$ & 0.61 & $*$ \\
\hline
\end{tabular}

Table 8. Simple correlation and regression values of the population dynamics of Siphoninus phillyreae and its parasitoids and predators on pomegranate trees in Assuit Governorate during 2011 season.

\begin{tabular}{|l|c|c|c|c|}
\hline Variable & Simple correlation "r" & Probability "P" & Regression & Probability "P" \\
\hline Encarsia inaron & 0.71 & $*$ & 0.66 & $*$ \\
\hline Maximum & 0.73 & $* *$ & 0.70 & $* *$ \\
\hline Minimum & 0.68 & $* *$ & 0.64 & $* *$ \\
\hline R.H. \% & 0.60 & $*$ & 0.58 & $*$ \\
\hline
\end{tabular}


Table 9. Simple correlation and regression values of the population dynamics of Siphoninus phillyreae and its predators on pomegranate trees in Daqahlyia Governorate during 2010 season.

\begin{tabular}{|l|c|c|c|c|}
\hline Variable & $\begin{array}{c}\text { Simple } \\
\text { correlation "r" }\end{array}$ & Probability "P" & Regression & Probability "P" \\
\hline Clitostethus arcuatus & 0.42 & Ns & 0.33 & Ns \\
\hline Maximum & 0.70 & $* *$ & 0.67 & $* *$ \\
\hline Minimum & 0.61 & $* *$ & 0.59 & $* *$ \\
\hline R.H. \% & 0.55 & $*$ & 0.52 & $*$ \\
\hline
\end{tabular}

Table 10. Simple correlation and regression values of the population dynamics of Siphoninus phillyreae and its predators on pomegranate trees in Daqahlyia Governorate during 2011 season.

\begin{tabular}{|l|c|c|c|c|}
\hline Variable & $\begin{array}{l}\text { Simple } \\
\text { correlation "r" }\end{array}$ & Probability "P" & Regression & Probability "P" \\
\hline Clitostethus arcuatus & 0.40 & Ns & 0.37 & $\mathrm{~ns}$ \\
\hline Maximum & 0.81 & $* *$ & 0.82 & $* *$ \\
\hline Minimum & 0.72 & $* *$ & 0.70 & $* *$ \\
\hline R.H. \% & 0.68 & $*$ & 0.63 & $*$ \\
\hline
\end{tabular}




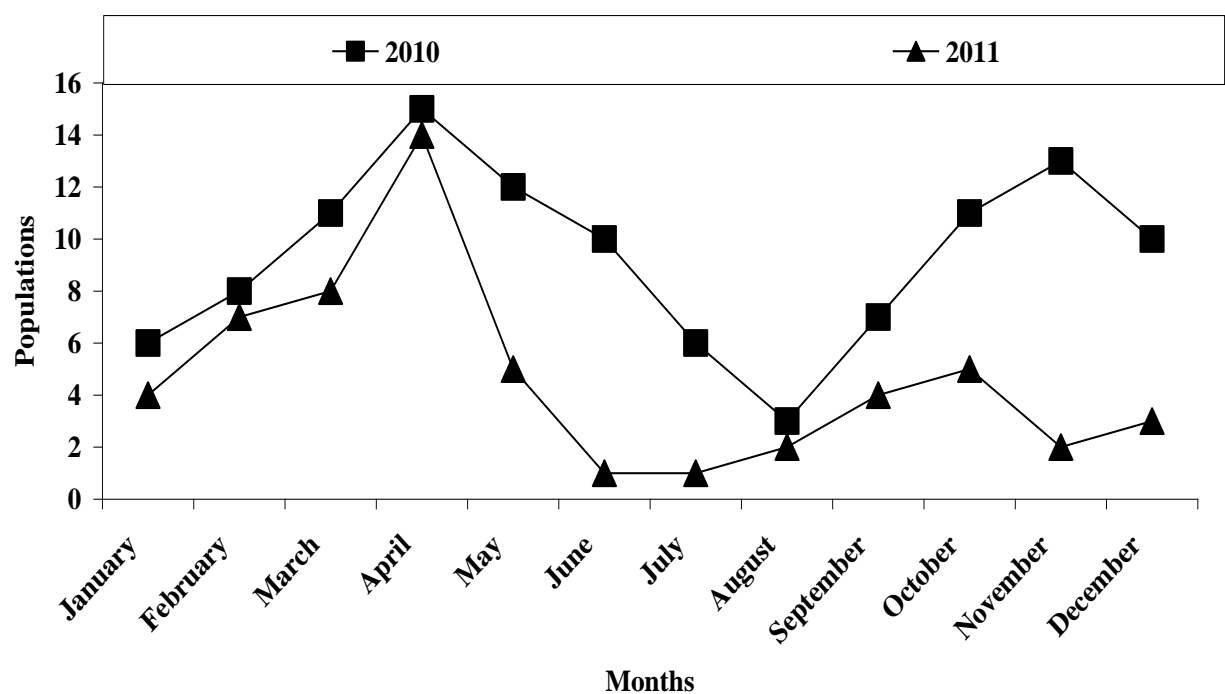

Fig. (1): Populations of Acaudaleyrodes rachipora on Citrus trees in Qalyubyia during 2010 and 2011

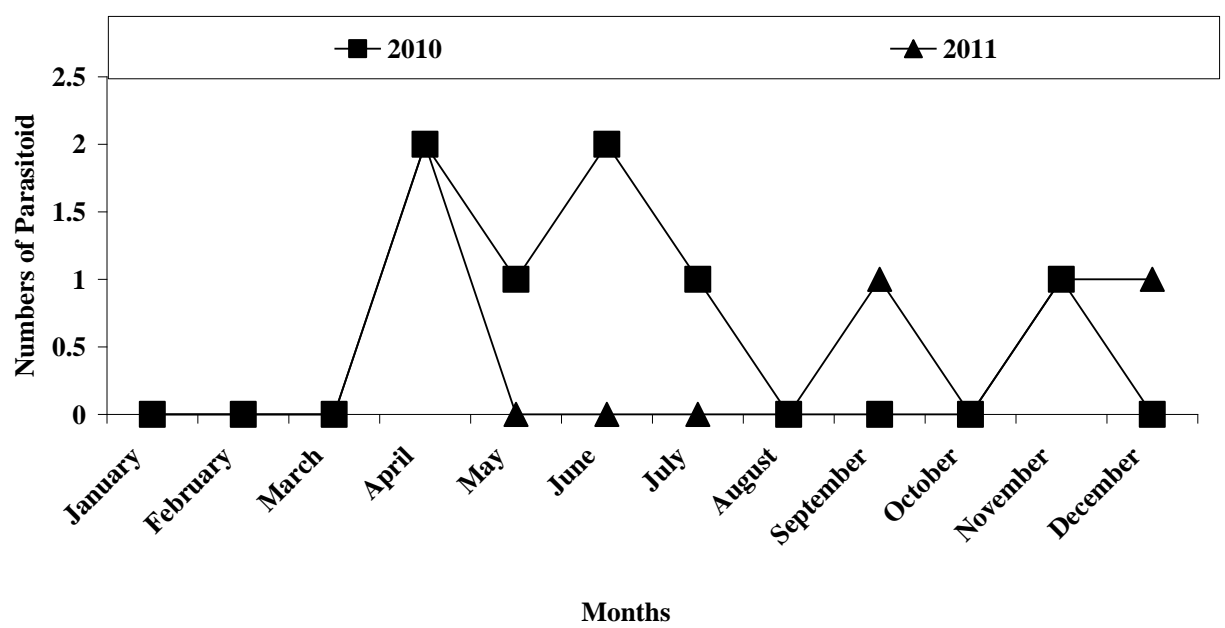

Fig. (2): Numbers of parasitoid, Encarsia acaudaleyrodis of Acaudaleyrodes rachipora on Citrus trees in Qalyubyia during 2010 and 2011 


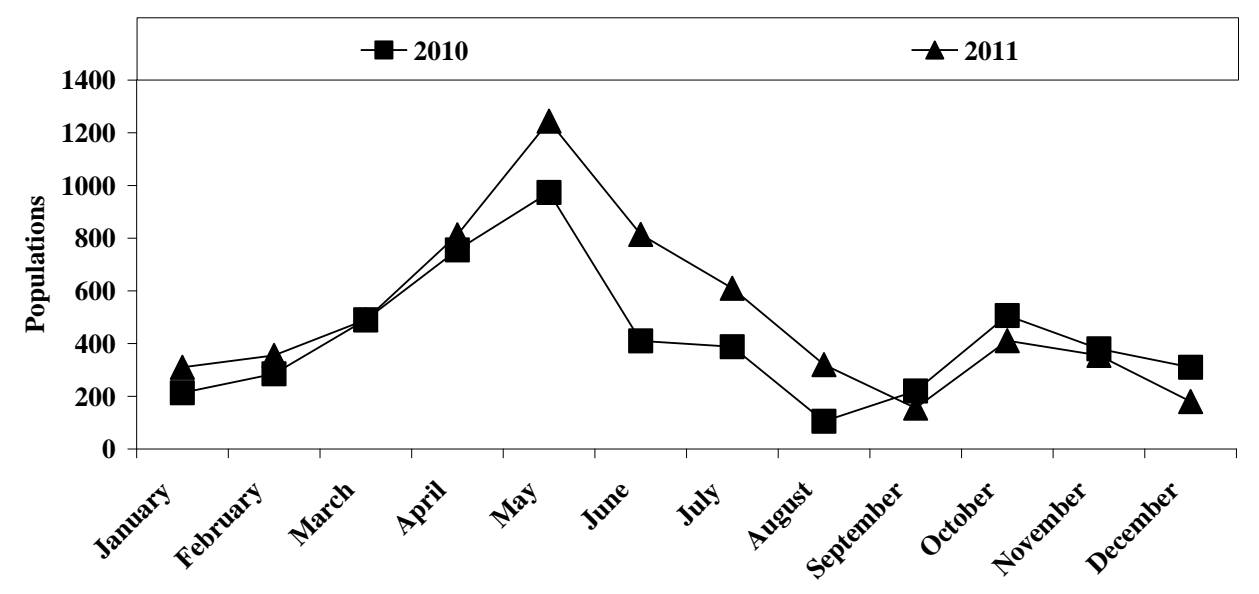

Months

Fig. (3): Populations of Aleurolobus marlatti on sycamore trees in Sharqyia during 2010 and 2011

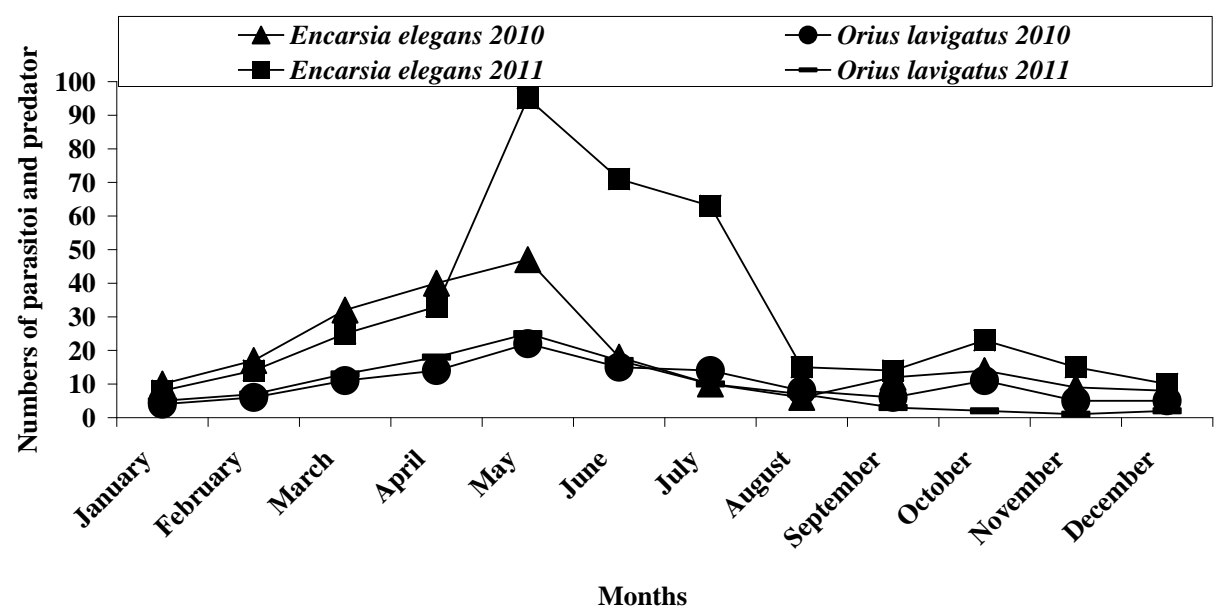

Fig. (4): Numbers of parasitoid, Encarsia elegans and predator, Orius lavigatus of Aleurolobus marlatti on sycamore trees in Sharqyia during 2010 and 2011 


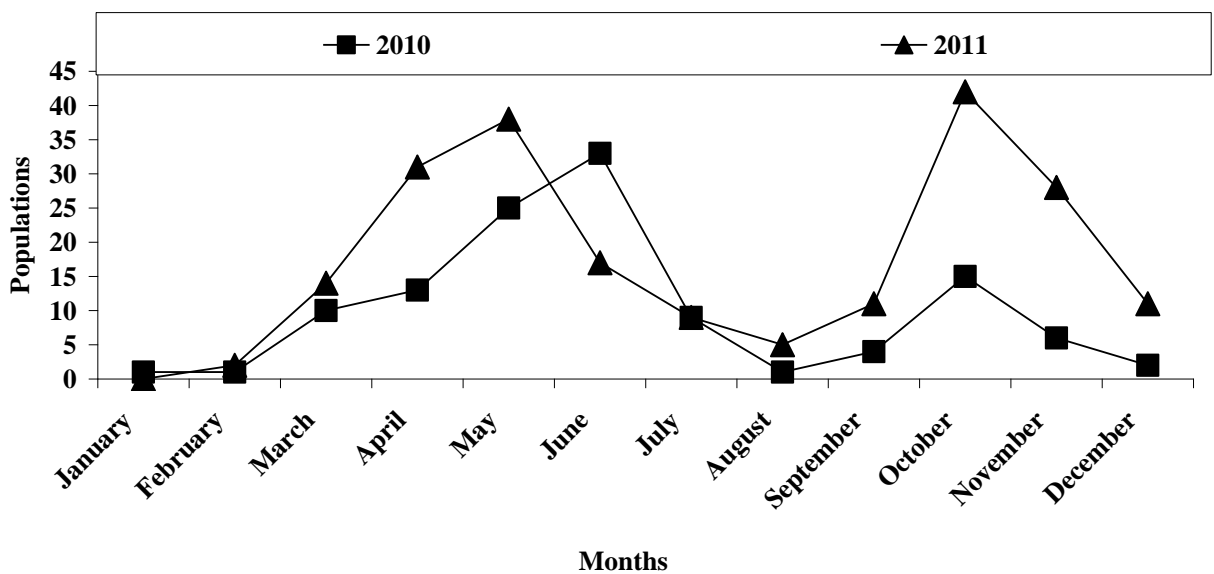

Fig. (5): Populations of Aleurolobus olivinus on olive trees in El-Arish during 2010 and 2011

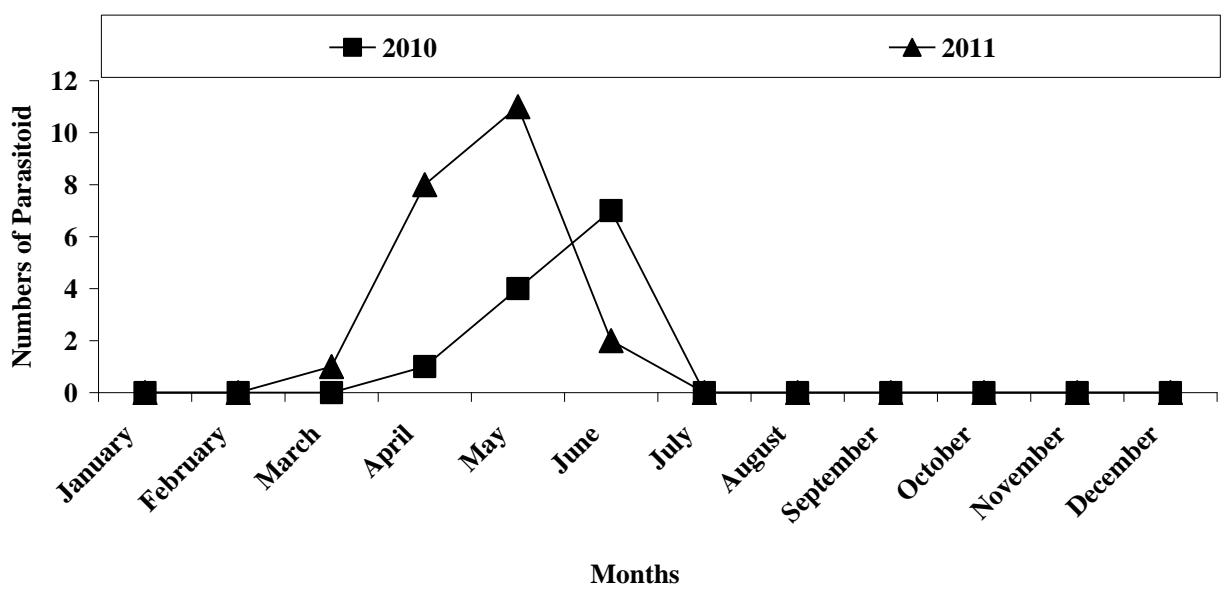

Fig. (6): Numbers of parasitoid, Encarsia olivine of Aleurolobus olivinus on olive trees in El-Arish during 2010 and 2011 


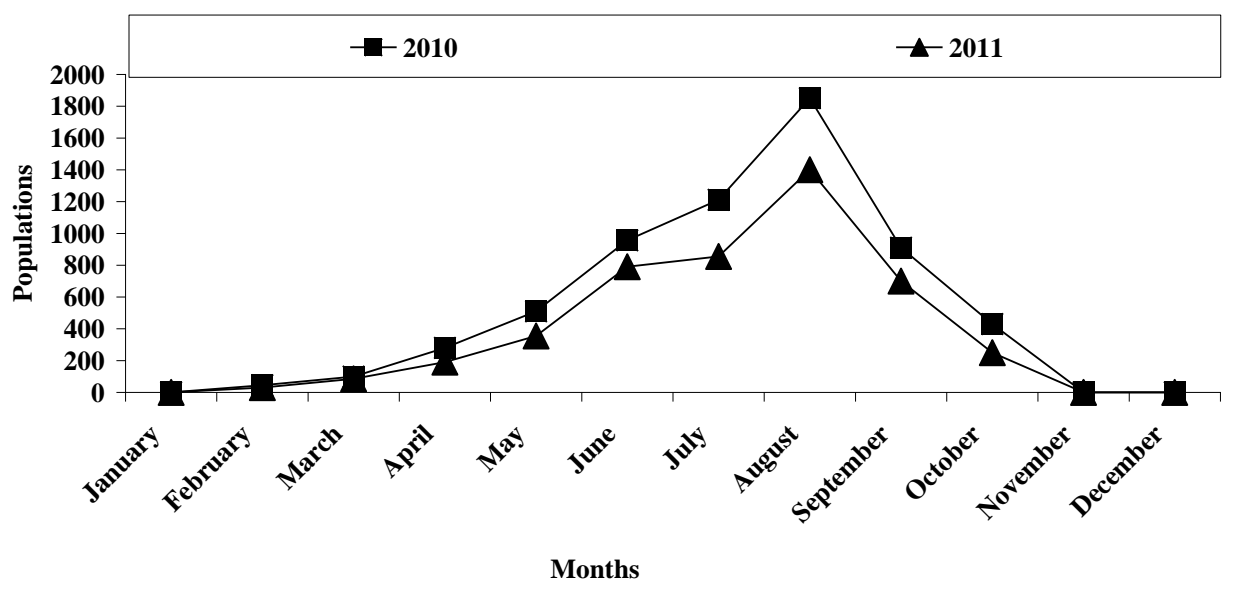

Fig. (7): Populations of Siphoninus phillyreae on pomegranate trees in Assuit during 2010 and 2011

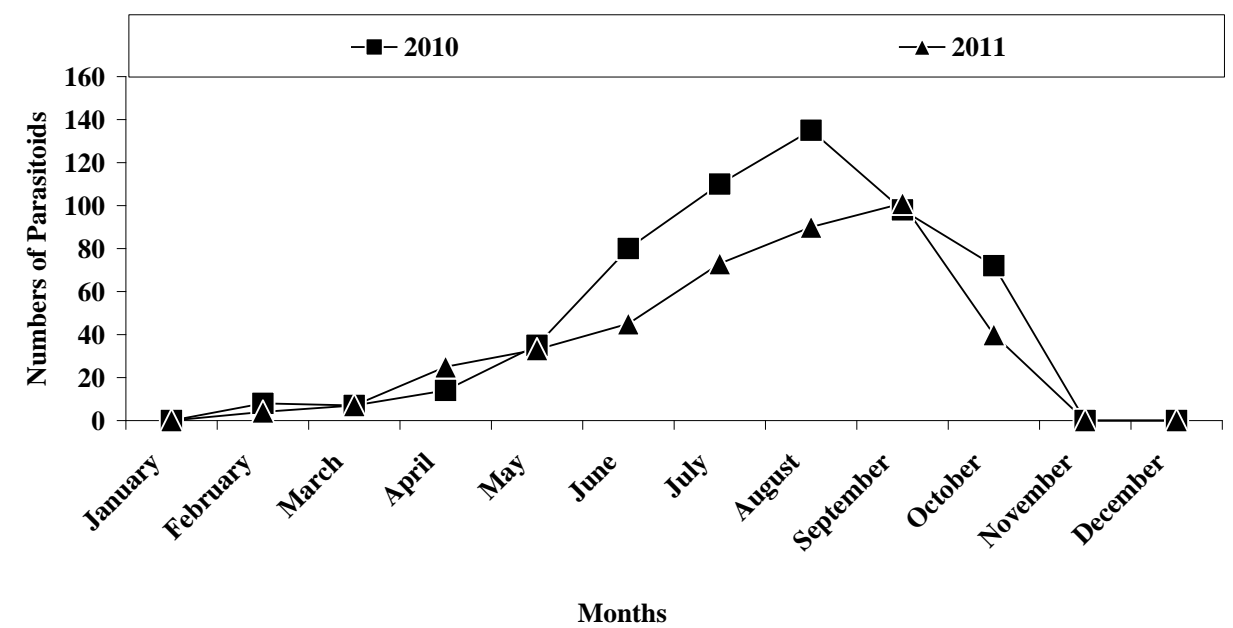

Fig. (8): Numbers of parasitoid, Encarsia inaron of Siphoninus phillyreae on pomegranate trees in Assuit during 2010 and 2011 


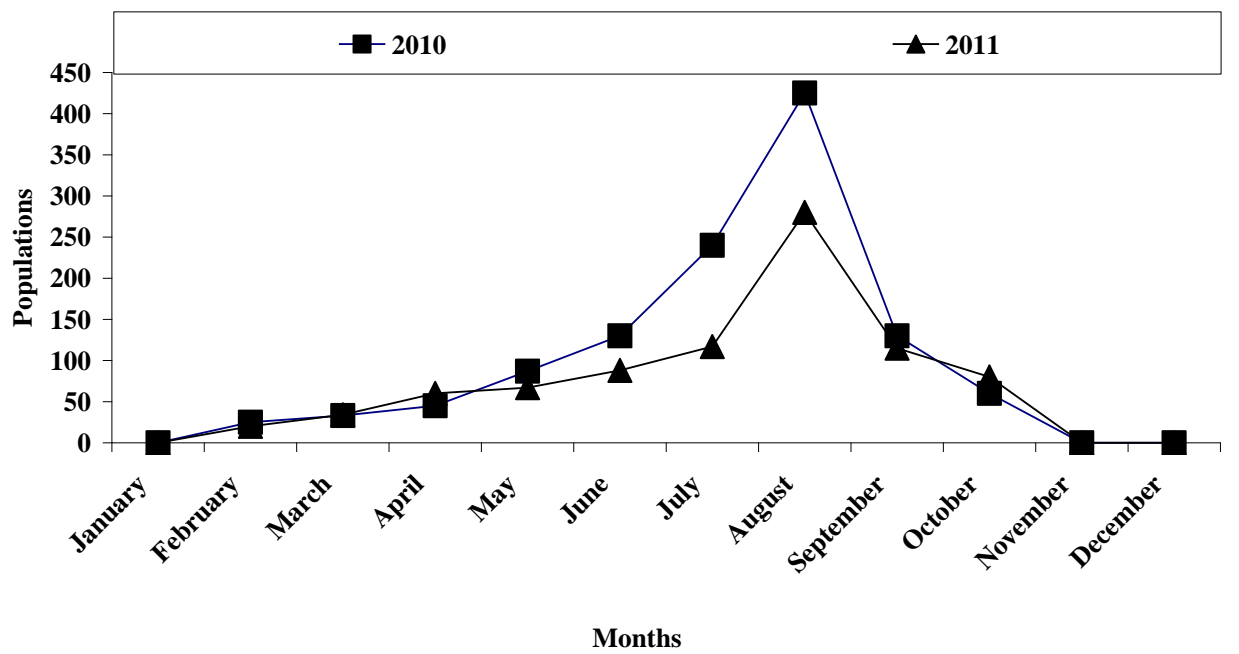

Fig. (9): Populations of Siphoninus phillyreae on pomegranate trees in Daqahlyia during 2010 and 2011

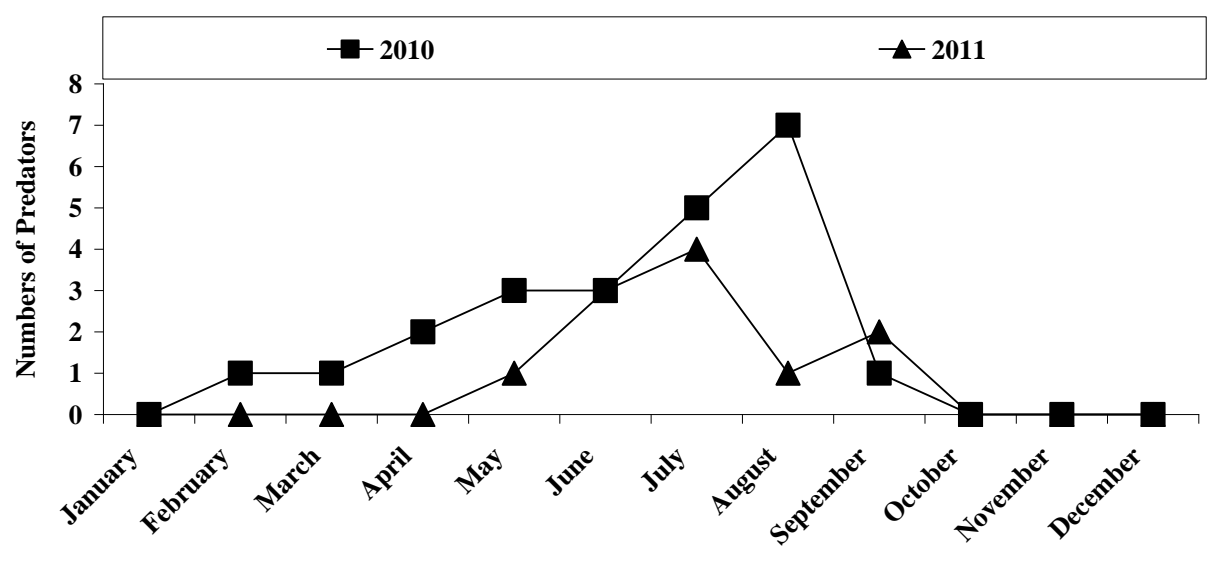

Months

Fig. (10): Numbers of predator,Clitostethus arcuatus of Siphoninus phillyreae on pomegranate trees in Daqahlyia during 2010 and 2011 


\section{REFERENCES}

1. Abd-Rabou, S. 1996. Egyptian Aleyrodidae. Acta Phytopathologica et Entomologica. Hungarica, 31 (3-4): 275-285.

2. Abd-Rabou, S. 1998. A revision of the parasitoids of whiteflies from Egypt. Acta Phytopathologica et Entomolgica Hungarica, 33 (1-2): 193-215.

3. Abd-Rabou, S. 1999. Successful introduction of Encarsia lahorensis Howard (Hymenoptera : Aphelinidae) for the control of Dialeurodes citri (Ashmead) (Homoptera : Aleyrodidae) in Egypt. Proc. of the first Regional Symposium for Applied Biological Control in Mediterranean Countries, pp. 35-38.

4. Abd-Rabou, S. 1999a. The efficacy of indigenous and imported predators utilized the biological control of Bemisia tabaci (Genn.) Biotype "B" (Homoptera : Aleyrodidae) in greenhouse. Acta Phytopathologica et Entomolgica Hungarica, 34 (4): pp, 333-339.

5. Abd-Rabou, S. 2006. Host plants, geographical distribution, and natural enemies of sycamore whitefly, Bemisia afer (Priesner and Hosny) (Homoptera: Aleyrodidae) as a new economic pest in Egypt. Fourth International Bemisia Workshop. Poster Presentation.

6. Abd-Rabou, S. 2006a. Biological control of the pomegranate whitefly, Siphoninus phillyreae (Homoptera: Aleyrodidae: Aleyrodinae) by using the bioagent, Clitostethus arcuatus (Rossi) (Coleoptera : Coccinellidae). Journal of Entomology, 3(4): 331-335.

7. Abd-Rabou, S. 2006b. Hymenopterous parasitoids as a bioagent for controlling homopterous insects in Egypt. Egypt. Adv. Agric. Res. In Egypt, Vol. (6) 1: 1-65.

8. Abd-Rabou, S. and A. Simmons. 2010. Augmentation and evaluation of a parasitoid, Encarsia inaron, and a predator, Clitostethus arcuatus, for biological control of the pomegranate whitefly, Siphoninus phillyreae. Archives of Phytopathology and Plant Protection,34(13): 1318-1334.

9. Abd-Rabou, S. 2011. Distribution and Key of Genus Bemisia Quaintance \& Baker in Egypt with Updating list of whiteflies in Egypt. Egypt. J.A.Res. ( Submitted).

10. Abd-Rabou, S. and M. Abou-Setta. 1998. Parasitism of Siphoninus phillyreae (Homoptera : Aleyrodidae) by aphelinid parasitoids at different locations in Egypt. J. of Hym. Res., 7 (1): 57-61.

11. Abd-Rabou, S. and A. M. Simmons. 2011. Survey of Reproductive Host Plants of Bemisia tabaci (Hemiptera: Aleyrodidae) in Egypt, Including New Host Records. Journal of Applied Entomology (Submitted) .

12. Evans, G. 2008. www.sel.barc.usda.gov:591/1WF/whitefly_catalog.htm - 14k . 
13. Habib, A. and F. A. Farag. 1970. Studies on nine common aleurodids of Egypt. Bull. Soc. Ent. Egypt, 54: 1-41.

14. Hafez, M.; M. F. S.; Tawfik, R. T. Awadallah and A. A. Sarhan. 1979. Natural enemies of the cotton whitefly Bemisia tabaci (Genn.) in the world and Egypt. Bull. Soc. Ent. Egypt, 62: 9-13.

15. (1979a).

Studies on Eretmocerus mundus Mercet, a parasite of the cotton whitefly Bemisia tabaci (Genn.) in Egypt. Bull. Ent. Soc. Egypt, 62: 15-22.

16. Mansour, K.M. 1995. Underutilized fruit crops in Egypt .CIHEAM OptionsMediterraneennes.P.13-19 http://ressources.ciheam.org/om/pdf/c13/96605636.pdf

17. Mesbah, A. H., A. H., El-Henidy, S. M. Metwally, Z. M. Shenishen and, H. S. Borael. 1999. Clitostethus arcuatus (Rossi) (Coleoptera: Coccinellidae), a new recorded predator species, of the citrus whitefly, Dialeurodes citri (Ashm.) (Homoptera: Aleyrodidae) in Egypt. Scientific Note. Egypt. J. Biol. Pest Cont. $8(2):$, p. $99-100$.

18. Papayiannis, L.C., N. Seraphides, Katis, N.I., M., Hadjist ylli, N. Loannon and J.K. Brown. 2008. Bemisia tabaci biotypes associated with whitefly transmitted virus epidemics in Cyprus. 3rd European whitefly symposium.

19. Priesner, H and M. Hosny. 1940. Notes on parasites and predators of Coccidae and Aleurodidae in Egypt. Bull. Soc. Ent. Egypt, 24: 58-70. 
الأباب الأبيض وأعدائه الحيوية على أشجار البساتين فى مصر

\section{شعبان عبد ريه و نها أحمد}

معهد بحوث وقاية النبات ـ مركز البحوث الزراعية ـ الدقي - الجيزة

يعتبر الذباب الأبيض من أهم الآفات الاقتصادية التي تتنتر على أثنار البساتين في

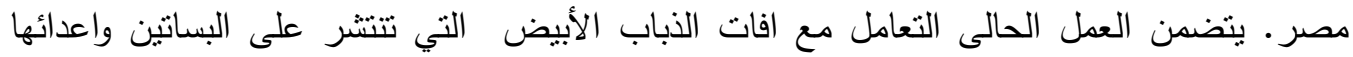

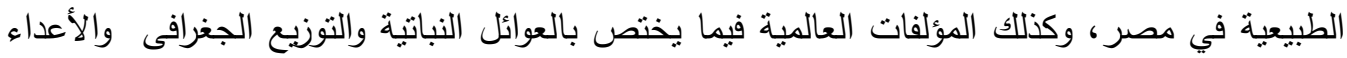

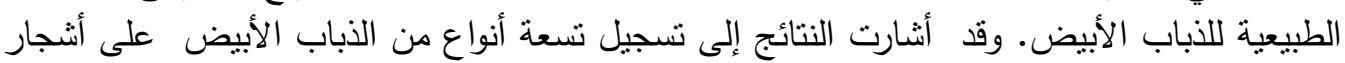

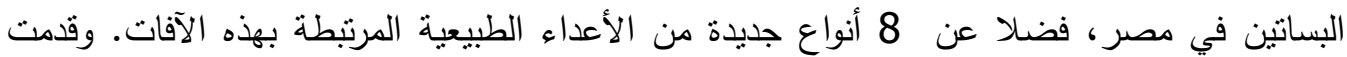

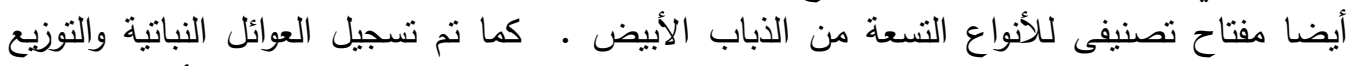

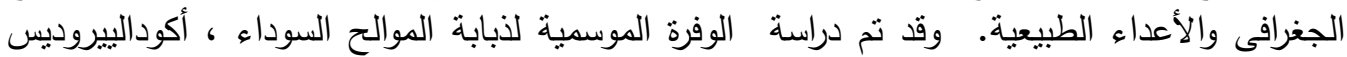

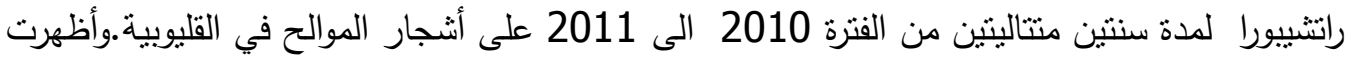

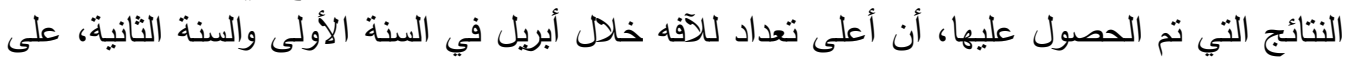

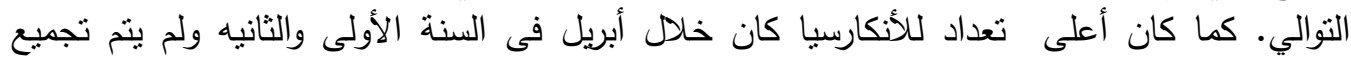

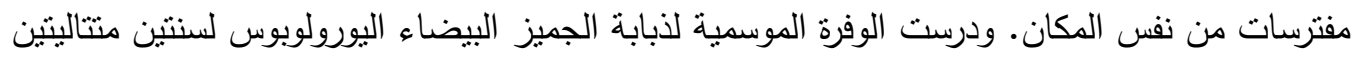

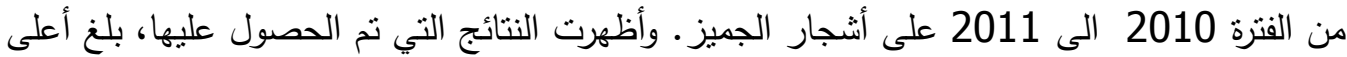

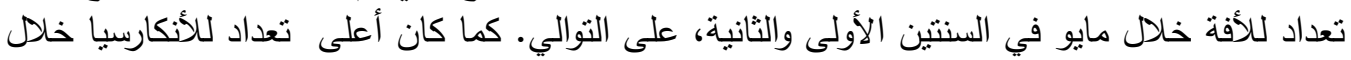
مايو فى السنة الأولى والسنة الثانية.

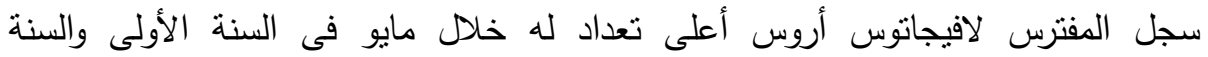

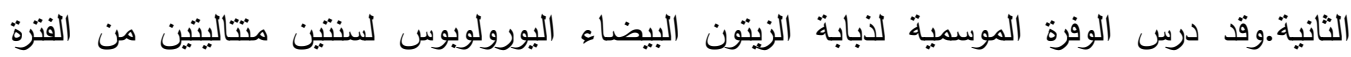

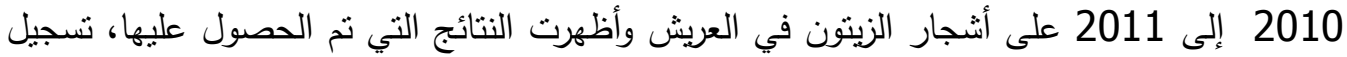

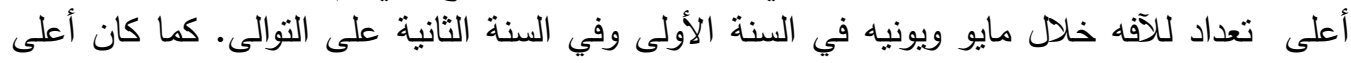

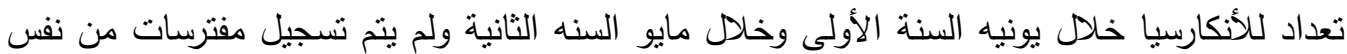

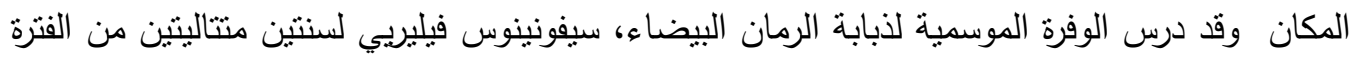

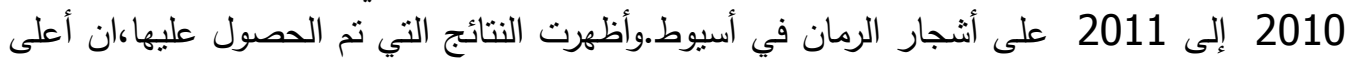

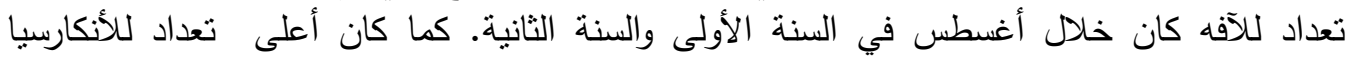

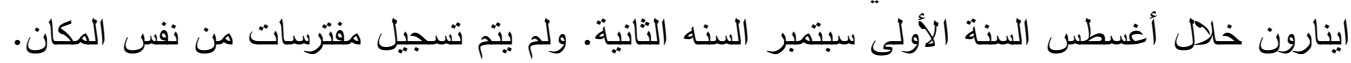
أيضا اجريت خلال نفس الدراسة سنتين متتاليتين من الفترة 2010 إلى 2011 على أنتجار الرمان

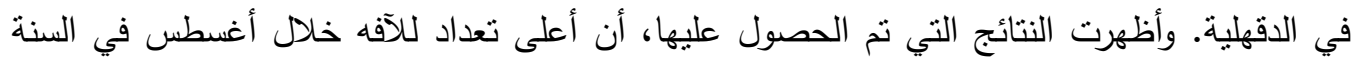

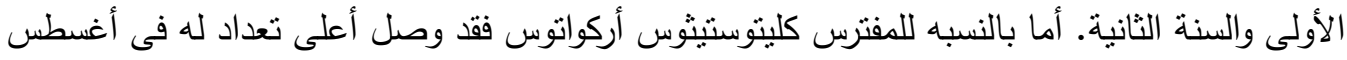

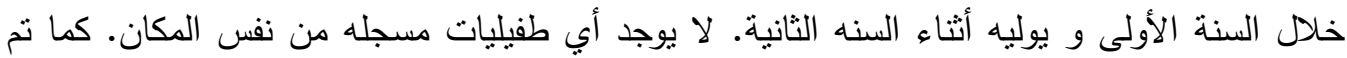

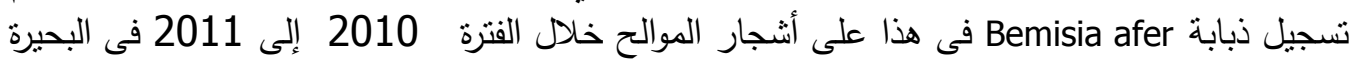

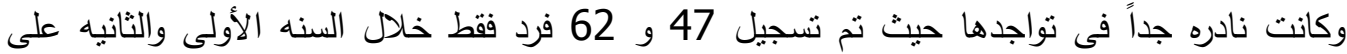

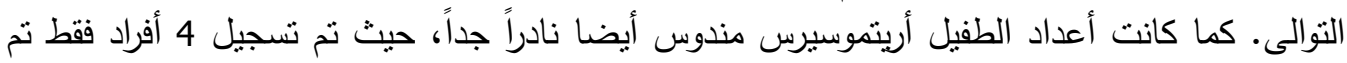

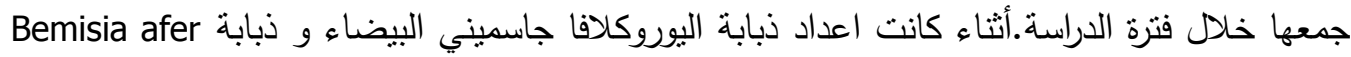

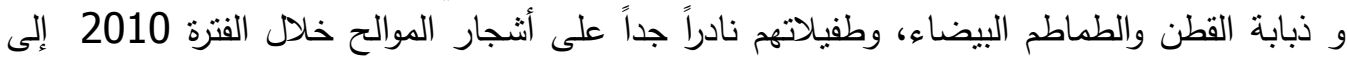

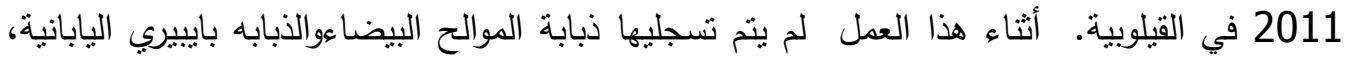
على أثنجار الموالح خلال الفترة 2011 إلى 2011 خلادل فترة الدراسة. 